\title{
A New Calibration Method for Robot Visual Measurement System without Handeye Calibration Procedure
}

\author{
Yali Wang \\ Electric and information engineering \\ Zhonghuan information college Tianjin university of \\ technology \\ Tianjin, china \\ wangyali_buaa@163.com
}

\author{
Yun Hao \\ Electric and information engineering \\ Zhonghuan information college Tianjin university of \\ technology \\ Tianjin, china \\ haoyun@tjzhic.com
}

\author{
Xihong Ye \\ Electric and information engineering \\ Zhonghuan information college Tianjin university of \\ technology \\ Tianjin, china \\ yexihong@tjzhic.com
}

\author{
Lu Yu \\ Electric and information engineering \\ Zhonghuan information college Tianjin university of \\ technology \\ Tianjin, china \\ yulu@tjzhic.com
}

\begin{abstract}
The relationship between the robot and visual measurement system (VMS) fixed on it is a critical part of the robot visual measurement system (RVMS). This paper presents a method to calibrate the robot and the VMS fixed on it without the traditional hand-eye calibration procedure. In the paper, the last robot link and the VMS are seen as a whole. The $Z$ axis of VMS is seen as the $Z$ axis of the robot last link frame. The kinematical model of the RVMS is created. According to the constraint that the world frame and the robot base frame are constant relatively, the objective functions are obtained, and the quantum behaved particle swarm optimization (QPSO) algorithm is used to calculate the unknown parameters. The results show that the precision of the calibrated system is $0.2 \mathrm{~mm}$ and is satisfied for large-scale workpiece measurement.
\end{abstract}

Keywords-robot visual measurement system; hand eye calibration; quantum behaved particle swarm optimization

\section{INTRODUCTION}

3D surface topography measurement and the aiming and positioning of the butt joint are key technology in large scale equipment manufacture and assemble process. With the developing of computer vision technology, visual measurement system as a kind of non-contact measurement method is used more and more widely. Besides noncontact, it has the advantages of high measurement precision and measurement speed. When the tested objects have fixed testing characteristics, and are mass produced, the framework structure is used. But with the increase of structure complexity and high machining precision requirement of mechanical parts, the demand to measurement is more and more strict. The framework structure cannot meet the measurement requirement. A flexible measurement system which can measure any part of the tested object is needed. Robot visual measurement system is a combination of robot and visual measurement system. Robot vision is seen to be the most important sensory ability for the robot [1]. Cameras fixed on the robot arm, allow it not only have the robot advantages of large flexible global motion, but also have the advantages of the visual measurement system.

For the robot visual measurement system, it is important to know the relationship between the robot and the camera. The traditional method computing the relationship is to calculate the transformation from the camera to the robot end-effector, which is called hand-eye calibration. The hand-eye calibration methods are mature [2,3], and most of them are solved by the equation $A X=X B$. Where $X$ is the hand-eye transformation matrix, $A$ is the robot forward kinematics, and $B$ is the camera extrinsic parameters. The rotation is calculated first, and then the translations are attained. There are at least six parameters need to calculated. In some researches [4], auxiliary measuring devices like auxiliary cameras or laser trackers are used to calibrate the hand eye relationship. Optimization algorithms are used to obtain more accuracy results [5]. But the results accuracy of most optimization algorithms relay on the initial values. The QPSO algorithm [6] can overcome this limitation and has been proved to have better optimization capability than other optimization algorithms [7].

In this paper, the VMS is seen as the last axis of the robot, and there are six parameters need to figure out. The QPSO algorithm is used to compute the unknown parameters.

This paper is organized as follows. The calibration principle will be presented in section 2. Experiment results will be shown in section 3 , and the conclusion will be provided in section 4 . 


\section{CALIBRATION PRINCIPLE}

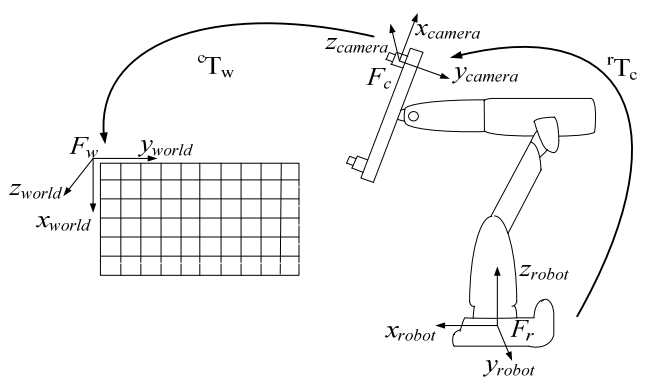

Figure 1. Robot visual measurement system calibration mode

As shown in Fig. 1, there is a VMS which consists of two cameras fixed on the robot end-effector. The right camera frame is seen as the VMS frame denoted by $F_{c}$. The world frame is created on the left top corner of the planar gridding target, and is denoted by $F_{w}$. The robot base frame $F_{r}$ and the world frame $F_{w}$ are relatively stationary. The VMS is moved to different positions with the robot, and the transformations ${ }^{r} T_{c}$ and ${ }^{c} T_{w}$ are changed at the same time. But the product of the two matrices is constant. Where, ${ }^{c} T_{w}$ is the transformation from the world frame $F_{w}$ to the VMS frame $F_{c}$, which is obtained by the binocular stereo vision system. ${ }^{r} T_{c}$ is the transformation from the VMS frame $F_{c}$ to the robot base frame $F_{r}$.

\section{A. Robot visual measurement system model}

The kinematic model of the robot is expressed by using Denabit-Hartenberg (DH) parameters [8]. The relationship between the robot end effector and the robot base frame can be expressed as (1). The VMS is fixed on the robot end-effector, and is seen as the sixth link of the robot. The RVMS model is shown in Fig. 2.

The kinematical parameters of the first five links can be obtained in reference [9], and the sixth link parameters are the unknown need to be calculated.

$$
\begin{aligned}
& A_{i}=\operatorname{rot}\left(Z_{i-1}, \theta_{i}\right) * \operatorname{trans}\left(Z_{i-1}, d_{i}\right) * \operatorname{trans}\left(X_{i}, a_{i}\right) * \operatorname{rot}\left(X_{i}, \alpha_{i}\right) \\
& =\left[\begin{array}{cccc}
C \theta_{i} & -S \theta_{i} & 0 & 0 \\
S \theta_{i} & C \theta_{i} & 0 & 0 \\
0 & 0 & 1 & 0 \\
0 & 0 & 0 & 1
\end{array}\right]\left[\begin{array}{cccc}
1 & 0 & 0 & 0 \\
0 & 1 & 0 & 0 \\
0 & 0 & 1 & d_{i} \\
0 & 0 & 0 & 1
\end{array}\right]\left[\begin{array}{cccc}
1 & 0 & 0 & a_{i} \\
0 & 1 & 0 & 0 \\
0 & 0 & 1 & 0 \\
0 & 0 & 0 & 1
\end{array}\right]\left[\begin{array}{cccc}
1 & 0 & 0 & 0 \\
0 & C \alpha_{i} & -S \alpha_{i} & 0 \\
0 & S \alpha_{i} & C \alpha_{i} & 0 \\
0 & 0 & 0 & 1
\end{array}\right] \\
& =\left[\begin{array}{cccc}
C \theta_{i} & -C \alpha_{i} S \theta_{i} & S \alpha_{i} S \theta_{i} & a_{i} C \theta_{i} \\
S \theta_{i} & C \alpha_{i} C \theta_{i} & -S \alpha_{i} C \theta_{i} & a_{i} S \theta_{i} \\
0 & S \alpha_{i} & C \alpha_{i} & d_{i} \\
0 & 0 & 0 & 1
\end{array}\right]
\end{aligned}
$$

Where $C \theta$ is the cosine value of the angle $\theta$, and $s \theta$ is the sine value of the angle $\theta$.
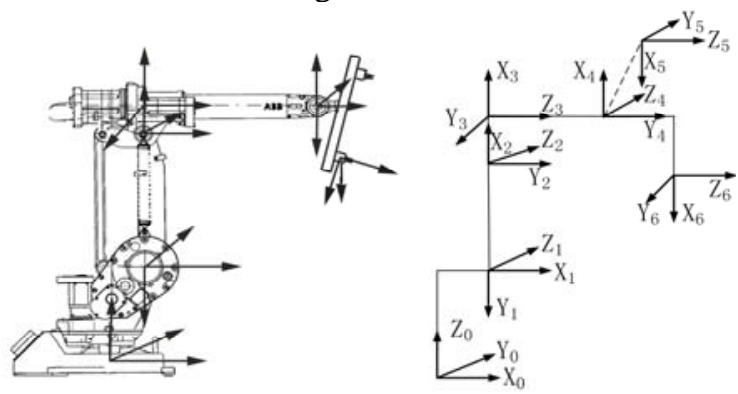

Figure 2. Robot visual measurement system model
For a minimal representation, the VMS frame relative to the fifth link frame can be denoted as a vector of three angles $(\alpha, \beta, \gamma)$ and a vector of three distances $(x, y, z)$. These angles are known as Euler angles when each represents a rotation about an axis of the VMS frame, and the angles are used to indicate $\mathrm{X}-\mathrm{Y}-\mathrm{Z}$ Euler angles in this paper. These distances are the translations between the two frames. As we all known that Euler angle representation of orientation always exhibits a singularity when $\beta= \pm 90^{\circ}$. But in this paper, $\beta<90^{\circ}$, so there isn't a singularity problem. According to the application, there are four steps to represent the transformation from the fifth link frame to the VMS frame.

As shown in Fig. 3, in step1, the VMS frame rotates about $X_{c 0}$ axis an angle of $-\alpha$. The VMS frame can be represented as $F_{c} X_{c 1} Y_{c 1} Z_{c 1}$. In step2, the new VMS frame rotates about $Y_{c 1}$ axis an angle of $\beta$. The VMS frame can be represented as $F_{c} X_{c 2} Y_{c 2} Z_{c 2}$. The $Z_{c 2}$ axis is still in the plane $F_{c} Z_{c 1} X_{c 1}$.

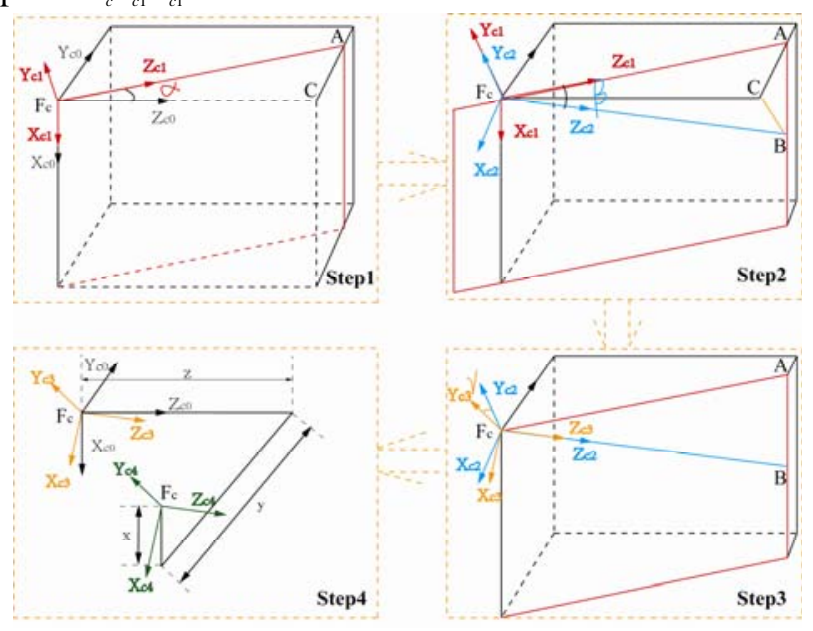

Figure 3. Transformation of the VMS frame and the fifth link frame

In step3, the VMS frame rotates about $Z_{c 2}$ axis an angle of $\gamma$. The VMS frame can be represented as $F_{c} X_{c 3} Y_{c 3} Z_{c 3}$.In step4, the VMS frame origin moves a distance of $-\mathrm{x},-\mathrm{y}$, and $\mathrm{z}$ from the fifth link frame origin along the $X_{5}$ axis, $Y_{5}$ axis and $Z_{5}$ axis separately. The VMS frame can be represented as $F_{c} X_{c 4} Y_{c 4} Z_{c 4}$.

According to the D-H model, the sixth link frame based on the VMS can be created in step2 of the figure 4, because the $Z_{c 3}$ axis is the same as the $Z_{c 2}$ axis. First the $Z_{6}$ axis is located along the $Z_{c 2}$ axis. Then the $X_{6}$ axis is located along the common normal between the $Z_{5}$ and $Z_{6}$ axes. The origin of the sixth link frame is created on the intersection point of the $Z_{6}$ and $X_{6}$ axes. The $Y_{6}$ axis can be obtained based on the right hand rule. Therefore the robot sixth frame can be created as shown in Fig. 4(a). 


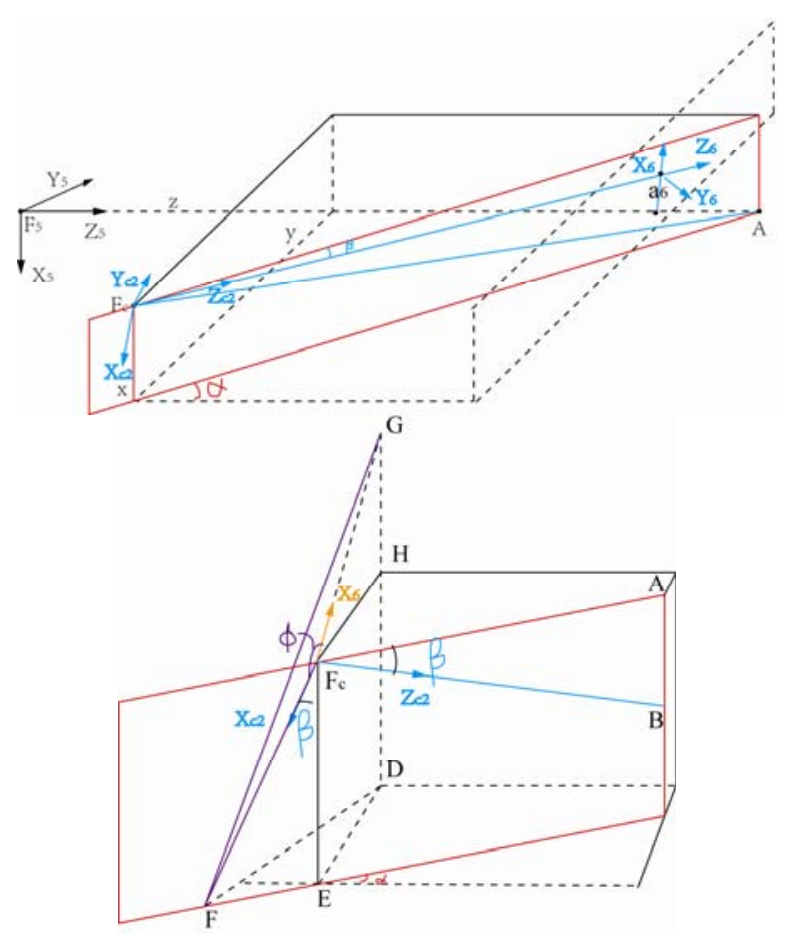

Figure 4.

Figure4(a) D-H model of the sixth link figure4(b) The procedure of computing $\phi$

Before the sixth link frame created, the common normal between the $Z_{5}$ and $Z_{6}$ axes should be obtained first. Suppose the coordinates of $F_{c} X_{c 0} Y_{c 0} Z_{c 0}$ is the origin, and the side-length of the cube is 1 . The direction of $Z_{5}$ axis is $\left[\begin{array}{lll}0 & 0 & 1\end{array}\right]^{T}$ and the point $p a=\left[\begin{array}{lll}0 & 0 & 0\end{array}\right]^{T}$ and point $p b=\left[\begin{array}{lll}0 & 0 & 1\end{array}\right]^{T}$ are on the line $Z_{5}$. The direction of $Z_{6}$ axis is $\left[\begin{array}{lll}A B & A C & 1\end{array}\right]^{T}=[\tan (\beta) / \cos (\alpha) \tan (\alpha) \quad 1]^{T}$, and point $p c=\left[\begin{array}{lll}x & y & z\end{array}\right]^{T}$ is on the line $Z_{6}$. The direction of common normal between the $Z_{5}$ and $Z_{6}$ axes is the cross of them. A plane can be determined by the line $Z_{5}$ and the common normal, and then the intersection point of the line $Z_{6}$ and the plane is the origin point of the sixth link frame, which denoted by $o p=\left[\begin{array}{lll}o x & o y & o z\end{array}\right]^{T}$.

The parameters of the sixth link are $\theta_{6}, \alpha_{6}, d_{6}$ and $a_{6}$. According to the definition of the $\mathrm{D}-\mathrm{H}$ model parameters, we have

$$
\left\{\begin{array}{c}
\theta_{6}=\theta+\pi-\arctan (\tan (\beta) / \sin (\alpha)) \\
d_{6}=o z \\
a_{6}=\sqrt{o x^{2}+o y^{2}} \\
\alpha_{6}=\arctan \left(\sqrt{(\sin (\alpha))^{\wedge} 2+(\tan (\beta))^{\wedge} 2} / \cos (\alpha)\right)
\end{array}\right.
$$

Where $\theta$ is the sixth link joint angle obtained from the robot angular encoder. In the paper, when the $\theta$ is not mentioned, it is seen as 0 default, which means the robot is on the initial situation.

From Fig. 4(a), we can see that the transformation from the VMS frame to the robot sixth frame has two steps.First the robot sixth frame rotates about $Z_{6}$ axis an angle of $\phi+\gamma$, and then translates a distance of $-L$ along $Z_{6}$ axis. Therefore the transformation can be written as

$$
{ }^{6} T_{c}=\left[\begin{array}{cccc}
C(\phi+\gamma) & -S(\phi+\gamma) & 0 & 0 \\
S(\phi+\gamma) & C(\phi+\gamma) & 0 & 0 \\
0 & 0 & 1 & -L \\
0 & 0 & 0 & 1
\end{array}\right]
$$

Where $L=\|o p-p c\|$ and

$$
\phi=-\arccos \left(-\sin (\alpha) / \cos (\beta) / \sqrt{\tan (\beta)^{\wedge} 2+\sin (\alpha)^{\wedge} 2}\right)
$$

The $\phi$ can be obtained from Fig. 4(b).

From Fig. 4(b), we can see that $E F=\tan (\beta)$, $\angle D E F=\pi / 2+\alpha$ and $E D=1$, so $D F$ can be obtained. $\angle G F_{c} H$ is known and $G H$ can be computed then $G D$ is obtained. So $G F$ is obtained. The angle $\phi$ can be obtained by $G F_{c}, F F_{c}$ and $G F$. Therefore the transformation from the VMS frame to the robot fifth frame can be written as

$$
{ }^{5} T_{c}={ }^{5} T_{6} *{ }^{6} T_{c}
$$

\section{B. Robot calibration}

According to the definition of the kinematical parameters of $\mathrm{D}-\mathrm{H}$ model, the twist angle describes the angle between two $Z$ axes of the adjacent links. When the robot is given, the twist angles are determinate. So the real $Z$ axes should be determined first to calibrate the twist angles. A laser tracker is used to measure the real $Z$ axes of the robot. The reflector is fixed on the robot endeffector, and the first axis of the robot is moved in the joint angle range uniformity. The motion curve of the reflector is a spatial circular arc, and the normal vector of the arc plane is the direction of the robot first link $Z$ axis. The same operations are performed to the rest links, and the directions of the $Z$ axes are obtained. But it is worth noting that there is a link gear between the link 2 and link 3 . When the link 2 is moved, the link 3 is moved at the same time. But it is not the same for the reverse condition. So when the operation is performed to the link 2, the reflector should be fixed on the arm of link 2, rather than on the robot end-effector.

The rest kinematical parameters of the robot are calibrated using the method mentioned in reference [10].

\section{The optimization algorithms}

Particle Swarm Optimization (PSO) is first introduced by Kennedy and Eberhart [11]. In the PSO algorithm, every swarm particle explores a possible solution. At first, the initial particles are generated in the searching space randomly. Then the particles as their personal best positions are used to evaluate the fitness function determined by the optimization problem. And the best position of the whole flock is the global best solution. Then the swarms adjust their own velocities and positions dynamically based on the personal best positions and global best positions.

But the particles will be trapped into local optima after several iterations. To make sure the particles escape from a local minimum, QPSO algorithm is proposed. In the QPSO algorithm, the particle position is described not by the velocity but by the particle's appearing probability density function. There is no fixed orbit for the particles, and the particles constrained by $\delta$ potential trough can appear at any position in the feasible solution space with certain probability. So the QPSO can guarantee the global convergence and it has been proved by Sun [6].

The particle position updating Equations are as follows 


$$
\begin{gathered}
C_{j}=\left(C_{1}, C_{2}, \ldots C_{D}\right)=\frac{1}{M} \sum_{i=1}^{M} P_{i}=\left(\frac{1}{M} \sum_{i=1}^{M} P_{i, 1}, \frac{1}{M} \sum_{i=1}^{M} P_{i, 2}, \ldots \frac{1}{M} \sum_{i=1}^{M} P_{i, D}\right) \\
p_{i, j}=\phi \cdot P_{i, j}+(1-\phi) \cdot G_{i, j} \\
x_{i, j}=p_{i, j} \pm \alpha \cdot\left|C_{j}-x_{i, j}\right| \cdot \ln [1 / u]
\end{gathered}
$$

Where, $\phi \sim U(0 \sim 1), u \sim U(0 \sim 1)$; and $D$ is the dimension of the problem space; $M$ is the population size; $x_{i}=\left(x_{i 1}, x_{i 2,} \ldots x_{i D}\right)$ and $P_{i}=\left(P_{i, 1}, P_{i, 2}, \ldots P_{i D}\right)$ are the particle current positions and the personal best positions; $G_{i}=\left(G_{i, 1}, G_{i, 2}, \ldots G_{i D}\right)$ are the global best positions; $\alpha$ is the ContractionExpansion coefficient which is the only parameter in QPSO algorithm depicted as

$$
\alpha=0.5 *(\text { MAXITER }-t) / \text { MAXITER }+0.5
$$

Where, $t$ is the current iterative number, and the MAXITER is the maximum iterative number.

\section{Object function}

According to the constraint that the transformation from the world frame to the robot base frame is constant, the object function is created. The VMS is moved with the robot to different positions. The target images are captured by the VMS, and the corresponding joint angles of the robot are recorded at the same time. Therefore the ${ }^{r} T_{5}$ and ${ }^{c} T_{w}$ in different positions are obtained. Then we can have a constant equation

$$
{ }^{r} T_{w}={ }^{r} T_{c} *{ }^{c} T_{w}={ }^{r} T_{5}{ }^{5} T_{c} *{ }^{c} T_{w}
$$

Then the objective function can be created and the parameters of ${ }^{5} T_{c}$ can be obtained. The objective function can be written as

$$
F(\alpha, \beta, \gamma, x, y, z)=\sum_{i=1}^{n-1} \sum_{j=i}^{n}\left\|{ }^{r} T_{5}^{i}{ }^{*} T_{c}^{i} *^{c} T_{w}{ }^{i}-{ }^{r} T_{5}^{j} *{ }^{5} T_{c}^{j}{ }^{*} T_{w}{ }^{j}\right\|^{2}
$$

Where ${ }^{c} T_{w}{ }^{i},{ }^{r} T_{5}^{i}$ and ${ }^{5} T_{c}^{i}$ are the transformation from VMS frame to the world frame, the transformation from the fifth link frame to the robot base frame and the transformation from the VMS frame to the fifth link frame respectively at the $i$ th position of the robot.

\section{EXPERIMENTS}

The robot is an industrial robot IRB1400 from ABB. The VMS consists of two industrial cameras AVT F504B with $17 \mathrm{~mm}$ lens. The image resolution of the camera is $2452 * 2056$ pixels. The field of view is about $300 * 300 \mathrm{~mm}^{2}$, and the work distance is about $600 \mathrm{~mm}$. The planar gridding target has a precision of $5 \mu \mathrm{m}$. The measurement system is shown in Fig. 5.

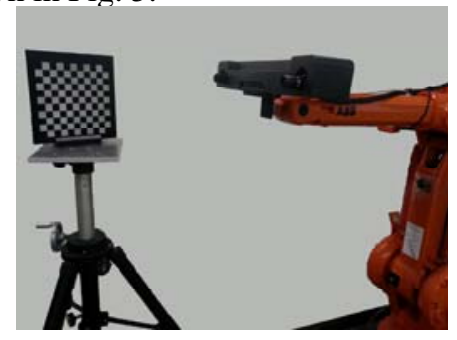

Figure 5. The robot visual measurement system

The two cameras are calibrated in advance. The right camera frame is the VMS frame $F_{c}$, and the left camera is an auxiliary camera to measure. The planar gridding target is placed in the robot work space, and the VMS is moved with robot to capture the images of the planar gridding target in different poses and positions.

The planar gridding target is placed at 5 different positions, and at each position, the cameras capture the images of the target at 10 different poses and positions. The calibration results are computed as follows

TABLE I. CALIBRATION RESULTS

\begin{tabular}{|c|c|c|c|}
\hline Parameter & Value & Parameter & Value \\
\hline$x$ & 42.5612 & $\alpha$ & 0.3516 \\
\hline$y$ & 191.3183 & $\beta$ & 0.0901 \\
\hline$z$ & 104.4643 & $\gamma$ & 0.0727 \\
\hline
\end{tabular}

The transformation of ${ }^{5} T_{c}$ can be written in matrix form as

$$
{ }^{5} T_{c}=\left[\begin{array}{cccc}
0.9933 & -0.0723 & 0.0900 & -42.5612 \\
0.0373 & 0.9386 & 0.3430 & -191.3183 \\
-0.1093 & -0.3374 & 0.9350 & 104.4643 \\
0 & 0 & 0 & 1.0000
\end{array}\right]
$$

To verify the performance of the proposed method, the standard rule with precision distance is measured using the calibrated robot visual measurement system. The distance of the target points on the standard rule is $1234.15 \mathrm{~mm}$. The standard rule is placed at 15 different positions and measured by the measurement system. The results are shown in table 2.

TABLE II. MEASUREMENT RESULTS

\begin{tabular}{|c|c|c|c|c|}
\hline No. & $\begin{array}{c}\text { Noncalibr } \\
\text { ated/mm }\end{array}$ & $\begin{array}{c}\text { Error/ } \\
\text { mm }\end{array}$ & $\begin{array}{c}\text { calibrated } \\
\text { /mm }\end{array}$ & $\begin{array}{c}\text { Error/ } \\
\text { mm }\end{array}$ \\
\hline 1 & 1237.2972 & 3.1472 & 1233.7635 & -0.3865 \\
\hline 2 & 1238.2079 & 4.0579 & 1233.9874 & -0.1626 \\
\hline 3 & 1230.4198 & -3.7301 & 1234.4825 & 0.3326 \\
\hline 4 & 1238.2837 & 4.1338 & 1234.6837 & 0.5338 \\
\hline 5 & 1235.4735 & 1.3236 & 1234.5175 & 0.3676 \\
\hline 6 & 1230.1254 & -4.0246 & 1234.6745 & 0.5246 \\
\hline 7 & 1231.9349 & -2.2150 & 1233.7785 & -0.3714 \\
\hline 8 & 1234.6188 & 0.4688 & 1234.7293 & 0.5793 \\
\hline 9 & 1238.7250 & 4.5751 & 1234.4971 & 0.3472 \\
\hline 10 & 1238.7988 & 4.6489 & 1234.6929 & 0.5430 \\
\hline 11 & 1230.7261 & -3.4239 & 1234.4561 & 0.3062 \\
\hline 12 & 1238.8559 & 4.7059 & 1234.6445 & 0.4945 \\
\hline 13 & 1238.7216 & 4.5717 & 1233.7637 & -0.3862 \\
\hline 14 & 1234.0037 & -0.1462 & 1234.5743 & 0.4244 \\
\hline 15 & 1237.1528 & 3.0028 & 1233.8869 & -0.2631 \\
\hline Mean/mm & 3.2117 & \multicolumn{3}{c|}{0.4015} \\
\hline RMS/mm & \multicolumn{3}{|c|}{11.2236} & \multicolumn{3}{c|}{} \\
\hline
\end{tabular}

\section{CONCLUSION}

This paper represents a robot visual measurement system calibration method considering the sixth link of the robot and the camera as a whole. The QPSO algorithm is used to compute the unkowns fast and precisely. The verified experiment results show the good performance of the proposed method. The proposed method has broad applications including numerous other kinds of hand eye calibration applications. The errors from the rotation of robot end effector have a big influence on the accuracy, so the robot should be calibrated before the proposed method is used.

\section{REFERENCES}

[1] Yuzhuo Zhong, Bingxin Qiao, Shuqing Li. Robot vision. National defense industry press.1994.

[2] Shiu Y C, Ahmad S. Calibration of wrist2mounted robotic sensors by solving homogeneous transform equations of the form $A X=$ 
XB[ J ]. IEEE Transactions on Robotics and Automation, 1989, 5(1) : 16 - 29.J.

[3] Yang Guang-lin, Kong Ling-fu, Wang Jie. A New Calibration Approach to Hand-Eye Relation of Manipulator. Robot .2006.28(4):400-405

[4] Suyi, L., and Guorong, W. Fast calibration for robot welding system with laser vision, Proceedings of the IEEE Conference on Robotics, Automation and Mechatronics,2008. pp 706-710.

[5] Strobl, K.H., and Hirzinger, G. Optimal hand-eye calibration, Proceeding of the IEEE/RSJ International conference on Intelligent Robots and Systems, Beijing,2006. pp 4647 - 4653.

[6] Sun J, Xu W, Liu J, Parameter selection of quantum-behaved particle swarm optimization. Advances in Natural Computation, 2005;3612: 543-552.

[7] Hassan R, Cohanim BK, Weck OD, et al. A comparison of particle swarm optimization and the genetic algorithm. In: Proceedings of the 1st AIAA Multidisciplinary Design Optimization Specialist Conference, April, 2005.

[8] Denavit J and Hartenberg RS. A kinematic notation for lower-pair mechanisms based on matrices. ASME J Appl Mechan 1955; 22: 215-221

[9] Product specification. Articulated robot 3HAC 9376-1.Rev.5 IRB 1400.

[10] Ren Yongjie, Zhu Jigui, Yang Xueyou, Ye Shenghua. Main Body Compensation Method of Flexible Measuring Robot for Car BIW. Opto-Electronic Engineering. 2009.Vol.36, No.2.

[11] Kennedy J and Eberhart RC. Particle swarm optimization. In: Proceedings of the IEEE International Conference on Neural networks, Perth, Western Australia, 1995, pp. 1942-1948, (IEEE Press, Piscataway, NJ). 